\title{
Localization of BRUNOL2 in Rat Spermatogenic Cells as Revealed by Immunofluorescence and Immunoelectron Microscopic Techniques
}

\author{
Hiroka Yonetamari, Yuko Onohara, Sadaki Yokota \\ Section of Functional Morphology, Faculty of Pharmaceutical Sciences, Nagasaki International University, \\ Sasebo, Nagasaki, Japan \\ Email: syokota@niu.ac.jp
}

Received March 8, 2012; revised April 12, 2012; accepted April 28, 2012

\begin{abstract}
Distribution and localization of a RNA-binding protein, BRUNOL2 in rat spermatogenic cells were studied by dot blotting of cell fractions, immunofluorescence (IF), and immunoelectron microscopy (IEM). BRUNOL2 distributed in nuclear $(23 \%)$, mitochondrial (19\%), microsomal (15\%), and cytosol fractions $(43 \%)$. BRUNOL2 was detected in all spermatogenic cells. In the cytoplasm and nucleoplasm of the spermatogonia, spermatocytes and spermatids, both diffuse and granular staining patterns were observed. Many cytoplasmic granules were stained also for DDX4 and DDX25. Large granules in the cytoplasm of elongated spermatids were stained for BRUNOL2 but not for the nuage proteins. IEM showed that gold signals for BRUNOL2 were concentrated in nuage components including loose aggregates of small particles, chromatoid body (CB), intermitochondrial cement (IMC), and satellite body (SB). In addition, many non-nuage structures such as ER-attached small granules, less dense material surrounding connecting piece of flagellum, reticulated body, mitochondria-associated granules (MAG), granulated body, ribosome aggregate, and manchette, were stained for BRUNOL2 with different staining intensities. In the nucleus, gold signals were concentrated in heterochromatin area and nucleolus. The results suggest that BRUNOL2 is one of the nuage proteins and also associated with the other non-nuage structures, suggesting multiple functions of this protein.
\end{abstract}

Keywords: BRUNOL2; Immunocytochemistry; Spermatogenesis; Nuage; Nucleus

\section{Introduction}

BRUNOL2 is a RNA-binding protein and belongs to CELF/BRUNO-like family. Its major function in the nucleus is a regulatory role in the alternative splicing of target pre-mRNAs and in the control of translation and mRNA stability in the cytoplasm [1-3]. CELF/BRUNOlike protein, CUGBP1, and the related protein Etr-3 regulate the muscle-specific splicing of cardiac troponin $\mathrm{T}$ pre-mRNA [4,5]. The binding of these proteins to CUG repeats suggests that they play roles in myotonic dystrophy $[4,6]$. CELF1/BRUNO-like protein is localized both in the cytoplasm and the nucleus [6-8]. This dual localization suggests multiple functions of the protein. In aged mouse liver, age-specific CUGP1-elF2 complex increases translation of CCAAT/enhancer-binding protein $\beta$ [9]. It has been shown that male $\operatorname{Cugbp1^{-/}}$ mice are infertile and the spermatogenesis is arrested at step 7 [10]. Thus, it has been suggested that BRUNOL2 plays crucial role in spermatogenesis but the function of BRUNOL2 is still unclear. Moreover detailed localization of BRUNOL2 in the spermatogenic cells has not been reported so far. In this paper, we study the co-localization of BRUNOL2 with known nuage proteins, DDX4 and DDX25, in rat spermatogenic cells by IF and IEM techniques.

We found that BRUNOL2 was localized to the cytoplasm and the nuclei of all spermatogenic cells. In the cytoplasm, it concentrated in the nuage components, and in the nuclei, it is associated with heterochromatin and granulated component of nucleolus attached to XY body. Such broad localization suggests the multiple functions of BRUNOL2 in the spermatogenic cells.

\section{Materials and Methods}

\subsection{Animals}

Japanese white rabbits $(3-4 \mathrm{~kg})$ and male Wistar rats (180 - 220 g) were obtained from Kyudo Co. Ltd. (Tosu, Japan) and fed appropriate standard diets and water ad libitum until use. The animal experiments were performed in accordance with the guidance for Animal Experiments, Nagasaki International University Maintaining the Integrity of the Specifications. 


\subsection{Antibody}

Polypeptide (GMKRLKVQLKRSKNDSKPY) consisting of 19 amino acids at the C-terminus of mouse CELF-1 (BRUNOL2) was synthetized and to the N-terminus cysteine was added to make chemical binding to carrier protein. The polypeptide $(1 \mathrm{mg})$ was conjugated with egg albumin $(50 \mathrm{mg})$ by using $m$-maleimidobenzoyl- $N$-hydrosuccinimide ester. The conjugate was emulsified with Freund's complete adjuvant. Rabbits and rats were intracutaneously injected with $200 \mu \mathrm{g}$ and $30 \mu \mathrm{g}$ of peptide, respectively. The injections were carried out 4 times with 2-week interval, and 2 weeks after last injection blood was collected. The specific antibody was affinity-purified by peptide-coupled column. Rabbit antibody against mouse DDX4 and rat antibody against mouse DDX25 were prepared as previously [11]. Rabbit anti-rat IgG was prepared by immunization of rabbits with purified rat $\operatorname{IgG}$ and the specific antibody was purified by affinity column coupled with rat IgG. Alexa Fluor ${ }^{\circledR} 568$ or Alexa Fluor ${ }^{\circledR} 488$ conjugated goat anti-rabbit IgG or goat anti-rat IgG were obtained from Molecular Probes (Eugene, OR). HRP-labeled goat antibodies to rabbit $\operatorname{IgG}$ and rat $\operatorname{IgG}$ were purchased from DAKO Japan (Tokyo). Protein A-gold probe (15 nm gold) was prepared by the method of de Roe et al. [12].

\subsection{Western Blotting}

Rat testes were homogenized in $5 \mathrm{mM}$ MOPS-KOH buffer (pH 7.4) containing $0.25 \mathrm{M}$ sucrose, $1 \mathrm{mM}$ ethylenediaminetetraacetic acid, $1 \mathrm{mM}$ phenylmethylsulfonyl fluoride and a cocktail of protease inhibitors $(1 \mu \mathrm{g} / \mathrm{ml})$ including leupeptin, pepstatin, aprotinin, and antipain (medium A) using a Potter-Elvehjem homogenizer. Ten percent homogenate $(\mathrm{w} / \mathrm{v})$ was centrifuged at $800 \times g$ for 10 $\min$. The resulting supernatant was centrifuged at 10,000 $\times g$ for $20 \mathrm{~min}$ and the pellet (mitochondrial fraction) was suspended in a small volume of medium A. The supernatant was centrifuged at $100,000 \times \mathrm{g}$ for $60 \mathrm{~min}$ in a Beckman ultracentrifuge using a SW 41 swing rotor. The resulting pellets were suspended in medium $\mathrm{A}$ and used as the microsomal fraction, while the supernatant was used as the cytosol fraction. The cell fractions were stored at $-70^{\circ} \mathrm{C}$. Protein concentrations were determined by the bicinchoninic acid method (Pierce Chemical, Rockford, IL) using bovine serum albumin (BSA) as a standard. The protein concentrations of the fractions were adjusted to $1 \mathrm{mg} / \mathrm{ml}$, mixed with one volume of sample buffer for SDS-PAGE, and heated in boiling water for 5 $\mathrm{min}$. Testes of mice and guinea pigs $(200 \mathrm{mg}$ of wet weight each) were homogenized with $1 \mathrm{ml}$ of SDS-PAGE sample buffer containing 0.1 volume of $0.3 \mathrm{M}$ iodoacetamide and heated in boiling water for $5 \mathrm{~min}$. Ten micrograms of each sample were analyzed by western blotting.
Molecular mass of BRUNOL2 was estimated by prestained protein maker (Nippon Genetics Europe GmbH, Dueren, Germany).

\subsection{Dot Blot Analysis of BRUNOL2 Content in Cell Fractions of Rat Testis}

The cell fractions including mitochondria, microsomes, and cytosol were isolated from rat testis homogenate by differential centrifugation as described above. Nuclei were isolated by the method of Rickwood and Ford (1983). Briefly, the crude nuclear fraction $(800 \times g$ pellet) was suspended in medium A containing $1 \%$ Triton X-100 and centrifuged at $800 \times g$ for $10 \mathrm{~min}$. The resulting pellet was suspended in $2 \mathrm{ml}$ of medium A, mixed with $72.5 \%$ (w/v) metrizamide (Sigma-Aldrich Japan, Tokyo) in medium A without $0.25 \mathrm{M}$ sucrose and centrifuged at 10,000 $\times g$ for $20 \mathrm{~min}$ at $5^{\circ} \mathrm{C}$. The nuclei-rich pellicles at the surface of the metrizamide solution were collected, suspended in a 10-times volume of medium A, and then centrifuged at $6000 \times g$ for $10 \mathrm{~min}$. The pellet was suspended in $1.6 \mathrm{ml}$ of medium A. Each fraction was diluted by medium A. The diluted fractions $(90 \mu \mathrm{l})$ were mixed with $90 \mu \mathrm{l}$ of SDS-PAGE sample buffer and $20 \mu \mathrm{l}$ of 0.3 $\mathrm{M}$ iodoacetamide, treated in boiling water for $5 \mathrm{~min}$, and centrifuged at $10,000 \times g$ for $10 \mathrm{~min}$. The supernatants were diluted 200 -fold and $1-5 \mu 1$ of them was loaded onto PDVF membranes for immunoblotting. BRUNOL2 protein was visualized using combination of rabbit antiBRUNOL, HRP-labeled goat anti-rabbit IgG, and DAB reaction. Internal standard proteins, histone $\mathrm{H} 2 \mathrm{~A}$ (nuclei), ICD1 (mitochondria), PDI (microsomes), and $\alpha$-tubulin (cytosol) were visualized using combination of rabbit antibodies against each protein, HRP-labeled goat anti rabbit $\operatorname{IgG}$, and $\mathrm{DAB}$ reaction. The staining intensity was measured using a densitometer. The total amount of BRUNOL2 in each cell fraction was calculated as follows; the obtained densitometric values were multiplied by the final volume of each fraction. The data were obtained from three measurements and the average values and standard deviation were plotted.

\subsection{IF Staining of Rat Testis}

Rat testes were extracted, embedded in Tissue Tek (Sakura, Japan), and frozen in isopentane cooled by liquid nitrogen. Frozen sections were cut into $8 \mu \mathrm{m}$ thick with a Leitz cryotome and fixed in $4 \%$ paraformaldehyde + $0.01 \% \mathrm{CaCl}_{2}+0.05 \mathrm{M}$ Hepes- $\mathrm{KOH}$ ( $\mathrm{pH} 7.4$ ) for $15 \mathrm{~min}$ at RT. After wash in PBS, sections were treated with 0.1 Triton X-100-PBS for $15 \mathrm{~min}$, followed by blocking with $2 \%$ fish gelatin-PBS. Sections were then incubated with anti-BRUNOL2 antibodies for $2 \mathrm{~h}$, followed by $1 \mathrm{~h}$-incubation with Alexa Fluor ${ }^{\circledR} 568$ conjugated goat anti-rabbit or rat IgG. For IF control, preimmune serum 
was used instead specific antibodies, followed by Alexa Fluor ${ }^{\circledR} 568$ conjugated secondary antibodies. Some sections were stained for DDX4 or DDX25 with combination of the primary antibodies and Alexa Fluor ${ }^{\circledR} 488$ conjugated secondary antibodies. Nuclei were stained by DAPI. Sections were mounted by Mowiol-DABCO mixture and examined with a Nikon Eclipse E600 fluorescence microscope (Nikon, Tokyo, Japan). The images were merged using Adobe Photoshop ${ }^{\mathbb{B}} 7.0$ to visualize cell contours. Each stage of seminiferous tubules was judged by size and shape of nuclei stained by DAPI according to stages of the cycle illustrated by Russell and coworkers [13].

\subsection{IEM Staining of Rat Testis}

Rat testes were dissected, cut into small tissue block, and fixed in $4 \%$ paraformaldehyde $+0.2 \%$ glutaraldehyde in Hepes-KOH buffer ( $\mathrm{pH}$ 7.4) for $1 \mathrm{~h}$ at $4^{\circ} \mathrm{C}$. The tissue blocks were dehydrated in graded ethanol series at $-20^{\circ} \mathrm{C}$ and embedded in LRWhite, which was polymerized under UV light at $-20^{\circ} \mathrm{C}$. Thin sections of LR White-embedded testis tissues were cut with a diamond knife equipped with a Reichert Ultracut R, mounted on nickel grids, and incubated with affinity purified antibodies to BRUNOL2 $(1 \mu \mathrm{g} / \mathrm{ml})$ overnight at $4^{\circ} \mathrm{C}$. Preimmune sera were used instead of the primary specific antibody for the control experiments. Sections were contrasted and then examined with a Hitachi H7650 electron microscope (Hitachi, Tokyo, Japan) at acceleration voltage of $80 \mathrm{kV}$. Stage of seminiferous tubules and step of spermatids were judged according to stages of the cycle illustrated by Russell et al. [13].

\section{Results}

\subsection{Western Blotting}

Our antibodies developed three bands in Western blotting; molecular mass of a major band was $52 \mathrm{kDa}$, which was observed in all testicular cell fractions examined. Minor two bands had molecular masses of $55 \mathrm{kDa}$ and $73 \mathrm{kDa}$ (Figure 1(a)). The $55 \mathrm{kDa}$ band was observed in the cytosol fraction, which seemed to be one of BRUNOL2 isoforms, whereas $73 \mathrm{kDa}$ band was detected in the mitochondrial fraction. Analysis of cell fractions by dot blotting showed that the cytosolic fraction contained approximately $43 \%$ of total amount of BRUNOL2, followed by nuclear fraction $(23 \%)$, mitochondria (19\%) and microsomes $(15 \%)$ in sequence (Figure 1(b)). Marker proteins, histone 2A (H2A), isocitrate dehydrogenase (ICD1), protein disulfide isomerase (PDI), and $\alpha$-tubulin ( $\alpha$-Tub) were detected prominently in nuclear fraction, mitochondria, microsomes, and cytosol, respectively (Figure 1(b), Dot blot).

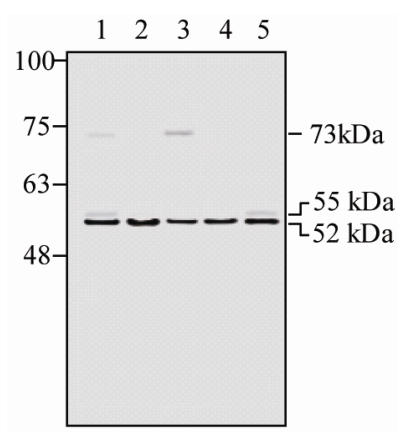

(a)

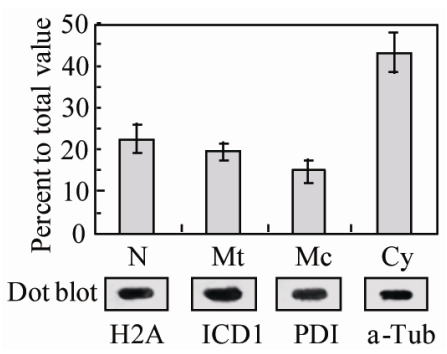

(b)

Figure 1. (a) Western blotting analysis of the cytosol fraction of rat testis homogenate. Lane 1: homogenate; lane 2: nuclear fraction; lane 3: mitochondrial fraction; lane 4: microsomal fraction, and lane 5: cytosol fraction. Rat anti-RRUNOL2 antibody developed three bands. Weakly stained 73 $\mathrm{kDa}$ band is detected in homogenate and mitochondrial fraction; $55 \mathrm{kDa}$ band is seen in homogenate and cytosol fraction; $52 \mathrm{kDa}$ band showing BRUNOL2 protein is developed in all cell fractions. (b) Dot blot analysis of cell fractions isolated from rat homogenate. $\mathrm{N}$ : nuclear fraction; Mt: mitochondrial fraction; Mc: microsomal fraction; Cy: cytosol fraction. Bars in each column are standard deviation. Dot blot shows marker protein of each cell fraction. H2A: histone H2A; ICD1: mitochondrial isocitrate dehydrogenase; PDI: protein disulfide isomerase; $\alpha$-Tub: $\alpha$-tubulin.

\subsection{IF Staining of Rat Testis for BRUNOL2}

Staining for BRUNOL2 was observed in the cytoplasm and nuclei of all spermatogenic cells with different staining intensities. Discrete granular staining varied greatly depending on developing stages. In stage I, small granular staining was noted in the cytoplasm of step 1 spermatids and pachytene spermatocytes. A single punctate staining was seen in the neck region of step 15 spermatids (Figure 2(a)). The nuclei of spermatids at steps 2-5 and pachytene spermatocytes at stages II-V were weakly stained. In the cytoplasm of spermatids at the same steps, irregularly-shaped large granules were stained, whereas the cytoplasm of pachytene spermatocytes at these stages was diffusely stained and contained a few granules (Figure 2(b)). In pachytene spermatocytes at stages V-IX, diffuse weak staining was observed in both nucleus and cytoplasm, but in elongated spermatids at step 9 numerous small granules appeared around nucleus (Figure 
2(e)). They disappeared soon; instead, slightly larger granules appeared in the cytoplasm of steps 10-11 spermatids (Figure 2(f)). In the residual body or cytoplasmic lobes of step 19 spermatids, round granules were stained (Figure 2(d)).

Next, we studied double staining of BRUNOL2 and DDX4. The results showed that there were three types of granules; large granules positive for both proteins (Figure 3), small granules positive for only BRUNOL2 located in the nuclei (Figure 3, arrows), and small granules stained for only DDX4 (Figure 3, circles). Moreover, fine granular staining identified as the intermitochondrial cement (IMC) in previous study [11] seemed to be buried in the
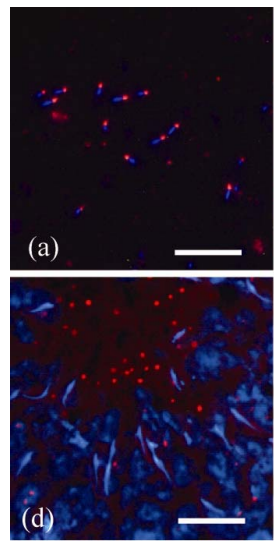
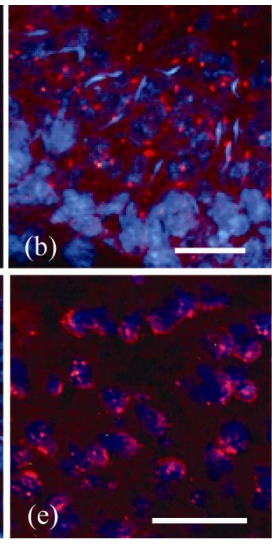
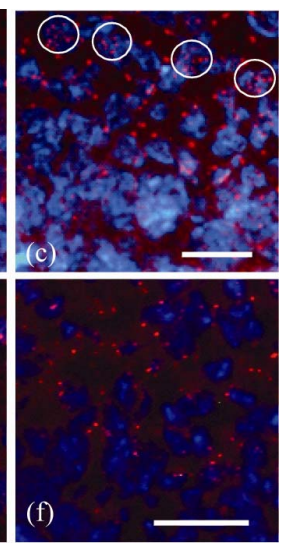

Figure 2. IF staining of BRUNOL2 protein in rat seminiferous tubule. Nuclei are stained with DAPI. (a) Step 15 spermatids. Note that a single punctate staining is present in the neck region of each spermatid; (b) Stage $V$ tubule. Large granules in spermatids are stained. The cytoplasm of spermatogonia, spermatocytes and spermatids is diffusely stained; (c) Stage VI tubule. Discrete granular staining is seen in the nuclei (circles) and cytoplasm of step 6 spermatids and diffuse staining is also in the cytoplasm of in spermatocytes and spermatids; (d) Stage VII tubule. Round granules in the cytoplasm of step 19 spermatids are stained; (e) Stage IX tubule. Fine granules along periphery of head of step 9 spermatids are stained; (f) Stage XI tubule. Small granules in the cytoplasm of step 11 spermatids are stained. Bar $=25 \mu \mathrm{m}$. strong cytoplasmic staining for BRUNOL2 (Figure 4). When the sections were stained doubly for DDX25 and BRUNOL2, large cytoplasmic granules with irregular shapes were stained for both proteins (Figure 5, arrows). The BRUNOL2-positive nuclear spots were not stained for DDX25 (Figure 5, circles). These staining reactions were not observed in the IF control sections incubated with pre-immune sera (Figures 6(a)-(c)).
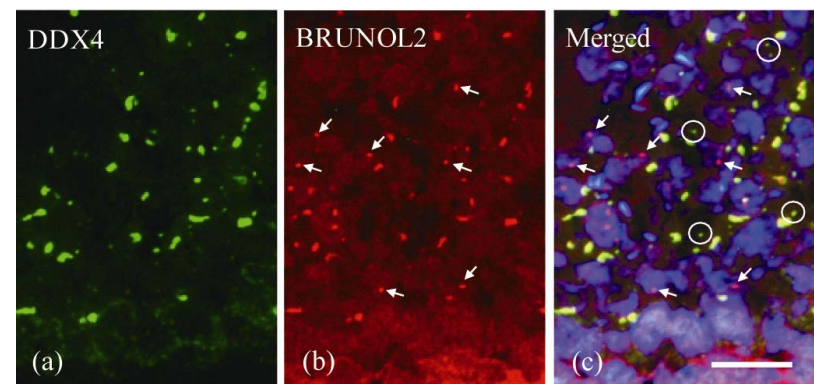

Figure 3. IF double staining of BRUNOL2 (red) and DDX4 (green) in seminiferous tubule at stage VI. (a) DDX4 staining; (b) BRUNOL2; (c) Merged (a) and (b). Stained spots in the nuclei of pachytene spermatocytes are negative for DDX4 (arrows) while small cytoplasmic granules are positive for only DDX4 (circles). The other large irregularly shaped granules are stained for both BRUNOL2 and DDX4. Nuclei are stained with DAPI. Bar $=25 \mu \mathrm{m}$.
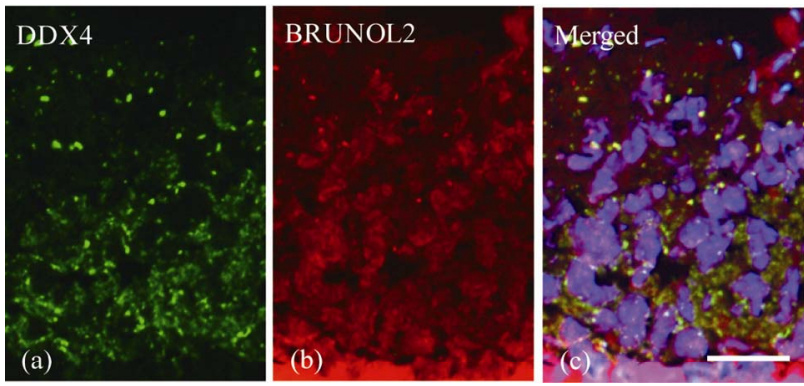

Figure 4. Localization of BRUNOL2 (red) and DDX4 (green) in seminiferous tubule at stage $X$. (a) Typical IMC contour is stained for DDX4; (b) BRUNOL2 is not stained in the IMC contour; (c) Merged (a) and (b). Nuclei are stained with DAPI. Bar $=25 \mu \mathrm{m}$.
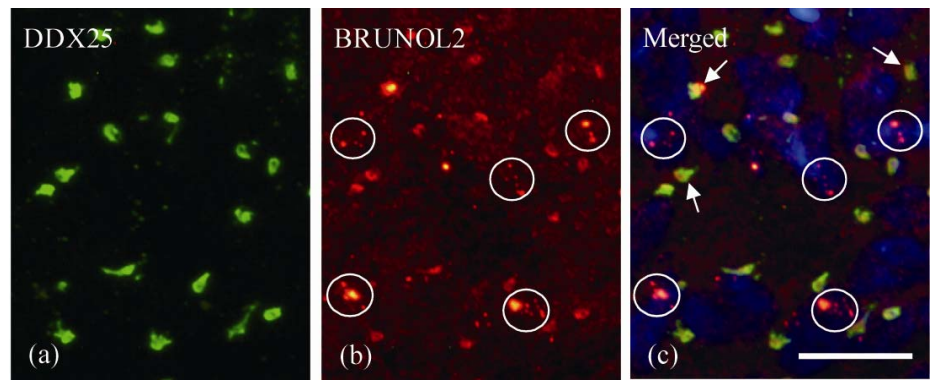

Figure 5. Localization of DDX25 (green) and BRUNOL2 (red) in rat seminiferous tubule at stage IV. (a) Granules with irregular shapes are stained for DDX25; (b) Staining for BRUNOL2; (c) Merged (a) and (b). Nuclei are stained with DAPI. Small spots are stained for BRUNOL2 (circles). A part of DDX25-positive large granules contain an area positive for BRUNOL2 (arrows). Nuclei are stained with DAPI. Bar $=25 \mu \mathrm{m}$. 


\subsection{IEM Localization of BRUNOL2 in Nuage and Other Compartments of Spermatogenic Cells}

Previously, we have studied the localization of DDX4 in rat spermatogenic cells and shown the protein is detected in the four types of nuage components [11]; 1) small particles appearing in meiotic cells, 2) intermitochondrial cement (IMC), 3) loose aggregates of small dense granules at the perinuclear area, and typical chromatoid bodies (CBs). Nuages of 1)-3) appear in spermatocytes and CBs occur in spermatids at steps 1 to 11 . In the present study, we focused on the localization of BRUNOL2 in these structures. Gold particles showing BRUNOL2 sites were localized to all the nuage components cited above but labeling intensity was essentially lower than that for
DDX4 [11]. Some of the small particles in meiotic cells were weakly stained (Figure 7(a)). Loose aggregates were stained intermediately, compared with the other nuage components (Figure 7(b)). IMCs were weakly or not stained for BRUNOL2 (Figure 7(c)). CBs were most strongly stained among the nuage structures (Figure 8(a)). They were sometimes connected with unstained materials (Figure 8(b)) and with satellite body (SB) which is slightly labeled (Figure 8(c)). The CBs migrate to the cytoplasm at the caudal side of the nucleus and decrease gradually in their volume. Such CBs were still stained for BRUNOL2 but the labeling intensity became lower than that of the CB (Figure 9(a)).

We have found the other BRUNOL2-positive structures, which were not classified into nuage, in the cyto-
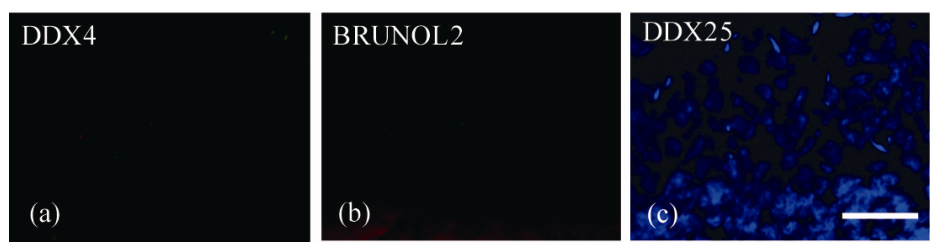

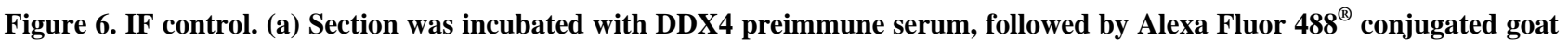
anti- rabbit IgG. No specific staining is observed; (b) Section was incubated with BRUNOL2 preimmune serum, followed by Alexa Fluor $568^{\circledR}$ conjugated goat anti-rat IgG. No specific staining is noted; (c) Section was incubated with DDX25 preimmune serum, followed by Alexa Fluor $488^{\circledR}$ conjugated goat anti-rat IgG. No specific staining is seen. Nuclei are stained with DAPI. Bar $=25 \mu \mathrm{m}$.
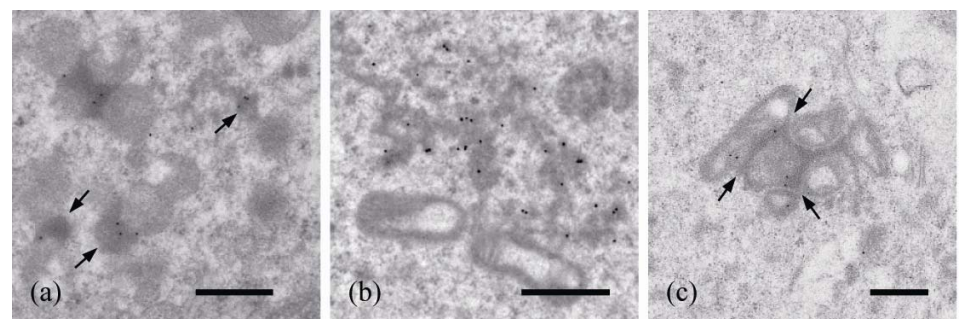

Figure 7. Localization of BRUNOL2 in various nuage components. (a) Small particles in meiotic cell are weakly labeled with gold particles (arrows). Some of them attach to mitochondria; (b) Loose aggregates of dense particles are stained; (c) Weak gold labeling is seen on IMC (arrows). Bar $=0.5 \mu \mathrm{m}$ for all.
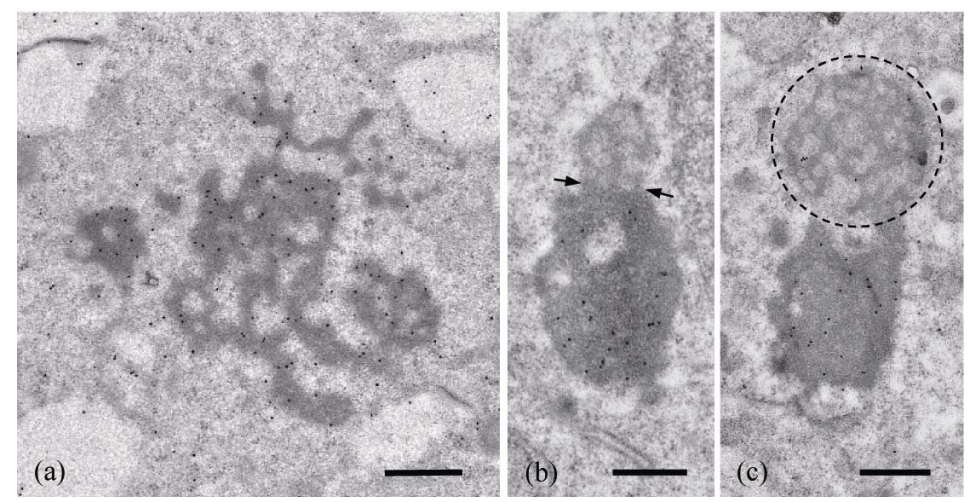

Figure 8. IEM localization of BRUNOL2 in chromatoid bodies (CBs) of spermatids. (a) Typical CB. Gold particles are seen on the dense material of CB; (b) CB connected with BRUNOL2-negative CB (arrows); (c) CB connected with weakly stained satellite body (circle). Bar $=0.5 \mu \mathrm{m}$ for all. 
plasm of spermatids at steps 6-7. They were small granules without the limiting membrane and closely associated with ER membrane (Figure 9(b)), and the material surrounding connecting piece between nucleus and flagellum (Figure 9(c)). BRUNOL2-positive structures were also observed in the cytoplasmic lobe of elongated spermatids at step 11-19. They were reticulated body in the cytoplasm of steps 14-16 spermatids (Figure 9(d)), mitochondria associated granules (MAG, Figure 9(e)), granulated body (Figure 9(f)), and large aggregates of free ribosomes (Figure 9(g)). The MAGs were appeared in the cytoplasmic lobe of the spermatids at steps 11-15 and relatively heavily labeled (Figure 9(e)). The granulated bodies were seen in the cytoplasm of step 14-15 spermatids and characterized by fine granular matrix and surrounding less dense small granules. Gold labeling was noted in the granular matrix (Figure 9(f)). The large aggregates of free ribosomes were one of the constituents of residual body of the spermatids at step 19. They were most strongly stained among the non-nuage components (Figure 9(g)). Furthermore, BRUNOL2 was weakly labeled in manchette of step 9 spermatids (Figure 9(h)). This labeling disappeared soon but no longer observed in the manchette of step 10 spermatids. No staining was noted in the nuage and the other structures cited above when the sections were incubated with preimmune serum instead with the primary antibody against BRUNOL2 (Figures 10(a)-(l)).

\subsection{IEM Localization of BRUNOL2 in the Nuclei of Spermatogenic Cells}

As shown by IF staining, BRUNOL2 was localized to the nuclei of spermatocytes and spermatids so that we addressed the localization of BRUNOL2 in the nuclei of differentiating spermatocytes and spermatids. The strong-

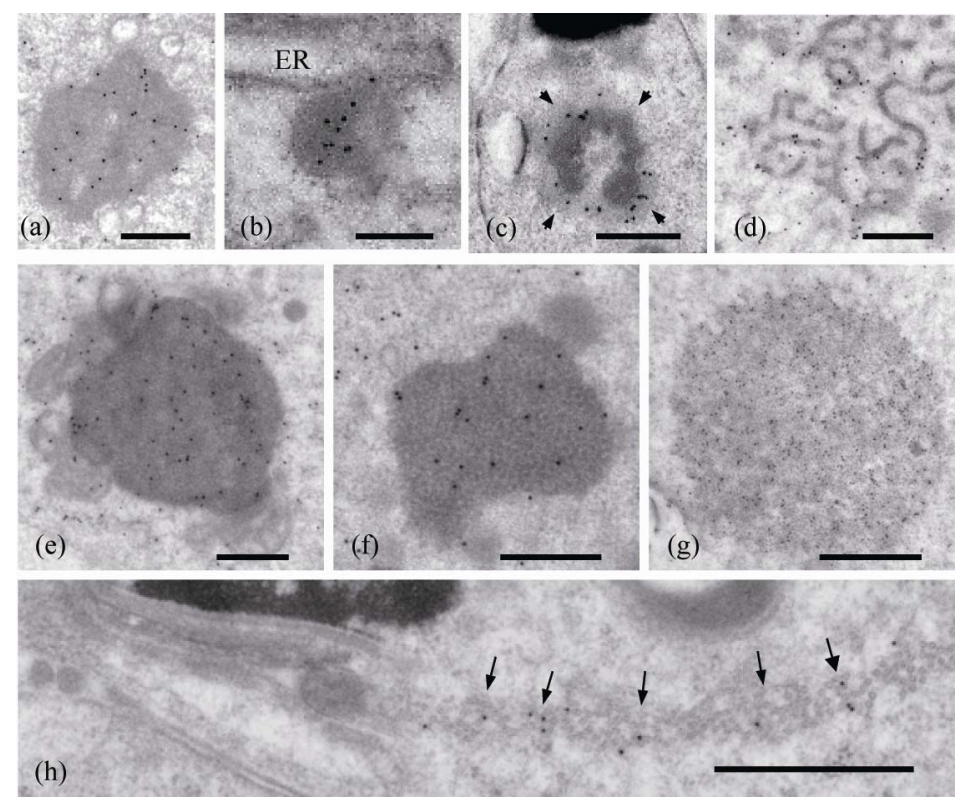

Figure 9. IEM localization of BRUNOL2 in various structures. (a) Late CB; (b) ER-attached small granule appearing in spermatids at steps 6-7; (c) Less dense material surrounding the connecting piece (arrows); (d) Reticulated body of step 15 spermatid; (e) Mitochondria associated granules (MAG); (f) Granulated body; (g) Aggregate of ribosomes; (h) Manchette of step 9 spermatid (arrows). Bar = $0.25 \mu \mathrm{m}$ for (b), $0.5 \mu \mathrm{m}$ for (a), (c), (d), (f), and (h), $1 \mu \mathrm{m}$ for (e) and (g).
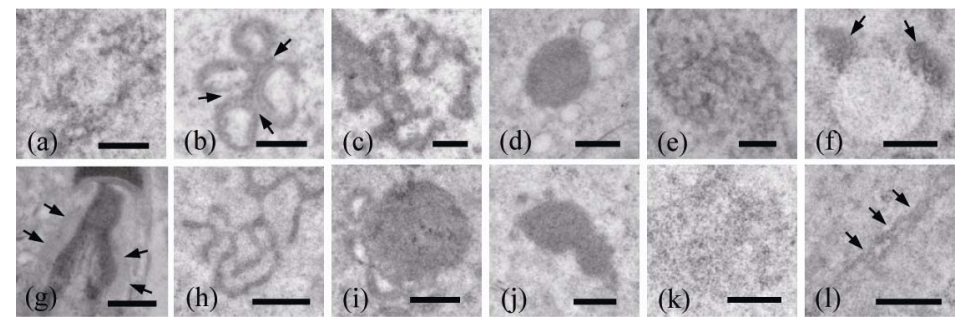

Figure 10. IEM control. Sections were incubated with BRUNOL2 preimmune serum, followed by incubation with rabbit antirat IgG and Protein A-gold probe (15 nm). (a) Loose aggregate; (b) IMC; (c) CB; (d) Late CB; (e) Satellite body; (f) ER attached small granules (arrows); (g) Material surrounding the connecting piece (arrows); (h) Reticulated body; (i) MAG; (j) Granulated body; (k) Ribosome aggregate; (l) Manchette (arrows). Bar = 1 um for all. 
est labeling was noted in the spermatocytes at stages IV VI. Heavy gold labeling was observed on the heterochromatin and the granular component of the nucleolus closely associated with the XY body (Figure 11). The euchromatin area was weakly or not labeled. The XY body was intermediately labeled (Figure 11). Gold labeling was observed in the nuclei of all spermatogenic cells including spermatogonia, spermatocytes and spermatids with different intensities (Figures 12(a) and (b)). The BRUNOL2 signals were associated with heterochromatin of these cells but not with euchromatin. BRUNOL2 was localized also to the nucleus of Sertoli cells in which the large nucleolus was strongly stained but the other area was weakly (Figures 12(c)). The nuclei of Leydig cells and other cells including fibroblasts, smooth muscle cells, endothelial cells of blood capillary, myoid cells were also labeled (data not shown). All these positive signals were not observed in the IEM control sections incubated with preimmune sera (data not shown). Summary of IEM results is shown in Table 1.

\section{Discussion}

\subsection{Distribution of BRUNOL2 Molecular Species in Rat Testicular Cell Fractions}

Three molecular species were shown by Western blotting. The $52 \mathrm{kDa}$ protein is BRUNOL2 and $55 \mathrm{kDa}$ and 73 $\mathrm{kDa}$ seem to be isoforms. The $73 \mathrm{kDa}$ molecule seems to be similar to isoform observed in heart, liver and brain [5] and is contained in the mitochondrial fraction by differential centrifugation. The $55 \mathrm{kDa}$ distributes solely in the cytosol. Present study showed that in rat testis $43 \%$ and $23 \%$ of total BRUNOL2 distributed in the cytosol and nuclear fractions, respectively. These results are largely consistent with those of IF and IEM staining. The nuclear fraction examined is confirmed to contain no CBs, many of which are precipitated into the nuclear fraction by usual differential centrifugal isolation, so that BRUNOL2 signals in the nuclear fraction are not due to contamination of CBs. Thus, the major sites of BRUNOL2 in spermatogenic cells of rat testis are cytosol and nuclei. BRUNOL2 in the mitochondria seems to be derived from coprecipitated CBs. BRUNOL2 detected in the microsomes might be due to the ER-attached small granules or membrane-bound ribosomes.

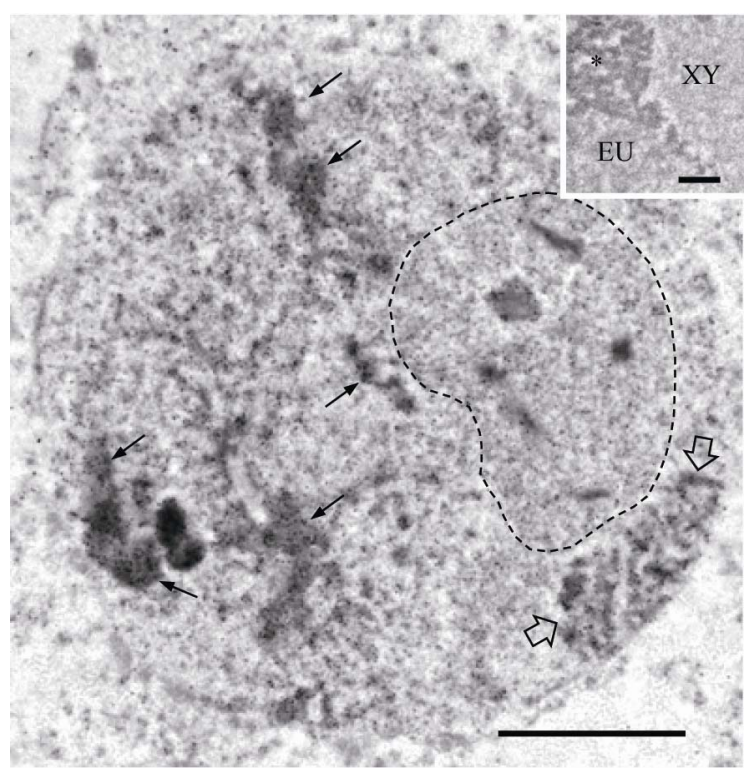

Figure 11. Localization of BRUNOL2 in the nucleus of a pachytene spermatocyte at stage VI. Heavy gold labeling is observed on dense heterochromatin (arrows) and the granular component of nucleolus (Large arrows). XY body surrounded by dashed line is intermediately stained. Euchromatin is weakly labeled. Inset. Control section incubated with BRUNOL2 preimmune serum. No gold signal is seen in XY body (XY), granular component of nucleolus (*), and euchromatin (EU). Bar $=2 \mu \mathrm{m}$ and $0.5 \mu \mathrm{m}$ for inset.
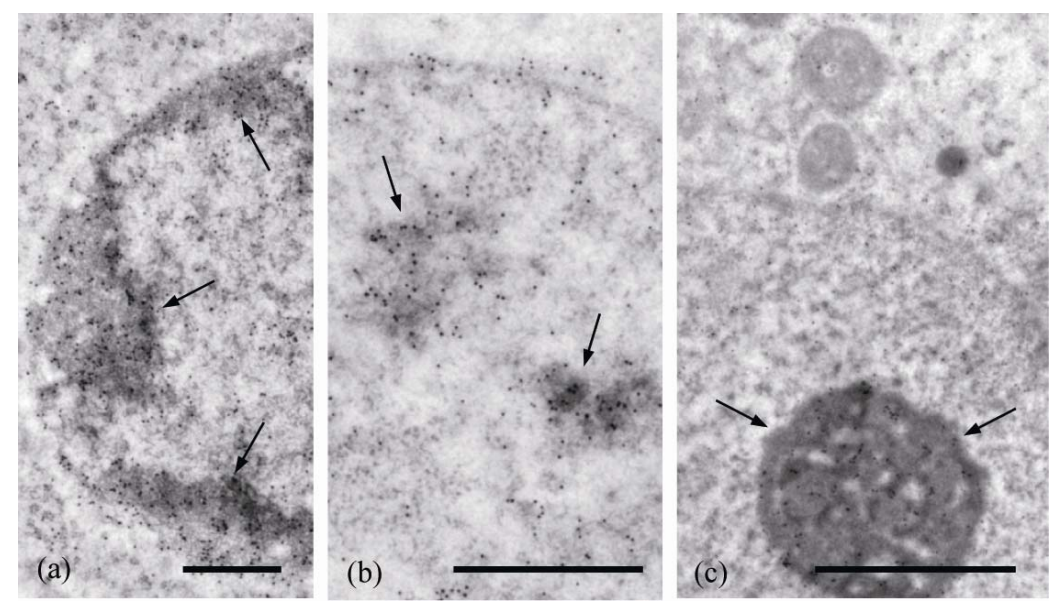

Figure 12. BRUNOL2 localization in the nucleus. (a) Spermatogonia. Heavy gold labeling is seen in heterochromatin (arrows); (b) Step 6 spermatid. Gold particles are concentrated on heterochromatin (arrows); (c) Sertoli cell. Gold labeling is seen on nucleolus (arrows). Bar $=1 \mu \mathrm{m}$. 
Table 1. BRUNOL2-positive structures in rat spermatogenic cells and their labeling intensity.

\begin{tabular}{llcl}
\hline Compartments & \multicolumn{1}{c}{ Structures } & Labeling intensity & \multicolumn{1}{c}{ Appearance } \\
\hline & IMC & +- & SPC \\
& Loose aggregate & ++ & SPC \\
& CB & ++ & SPT \\
& late CB & + & SPT \\
& SB & + & SPC and early SPT \\
Cytoplasm & ER-attached small granule & ++ & Steps 6-7 SPT \\
& Neck material & ++ & Steps 14-15 SPT \\
& Reticulated body & + & Steps 14-16 SPT \\
& MAG & ++ & Step 17 SPT \\
& Granulated body & ++ & Steps 14-16 SPT \\
& Ribosome aggregate & +++ & Step 19 SPT \\
& Manchette & + & Step 9 SPT \\
& Euchromatin & + All SPC and round SPT \\
& Heterochromatin & ++ & All SPC and SPT \\
& Nucleolus & +++ & All SPC and SPT \\
& XY body & + & Stage VII-IX SPC \\
\hline
\end{tabular}

SPC: spermatids, SPC: spermatocytes, +/-: weakly stained or not, + : weakly stained, ++ : intermediately stained, +++: strongly stained.

\subsection{Localization of BRUNOL2 in the Spermatogenic Cells}

If staining showed that BRUNOL2 distributed in both the cytoplasm and the nuclei of spermatogenic cells, including spermatogonia, all spermatocytes and spermatids. The nucleus of Leydig cells was also stained. The double staining revealed that BRUNOL2-positive irregularly shaped granules were completely overlapped with DDX4 and DDX25 staining. The results demonstrate that BRUNOL2 is localized in the CBs containing DDX4 and DDX25. In the cytoplasm, small numbers of granules were solely stained for BRUNOL2. Among these granules, those appearing in steps 6-7 spermatids seem to correspond to the ER-attached small granules observed by IEM. In addition, relatively large granules located in the cytoplasmic lobe of step 19 spermatids might be consistent with the aggregates of numerous free ribosomes shown by IEM. Spot staining in the neck region of the spermatids at steps 13-15 coincides with staining in the material surrounding connecting piece between nucleus and flagellum observed by IEM. Many punctate spots in the nuclei correspond to clustered heterochromatin, nucleoli, and XY body shown by IEM.

\subsection{Subcellular Sites for BRUNOL2 Revealed by IEM}

The present study showed clearly that BRUNOL2 was localized to nuage components classified previously by DDX4 distribution [11], such as CBs, late CBs, loose aggregates, IMC, and satellite body (SB). The results suggest that BRUNOL2 is functioning in these structures. However, the labeling intensity was consistently lower than that of DDX4 or DDX25. Especially, the labeling in the IMC was very weak or null, so that the major function sites of BRUNOL2 might be the CBs and the loose aggregates. Several proteins, such as DDX4 [11,14,15], DDX25 [16], TDRD1/MTR-1, TDRD6 and TDR7/TRAP [17,18], RNF17 [19], MIWI [20], kinesin KIF17b [20], Maelstrom [21] have been reported as nuage-constituent proteins so far. Among these proteins, RNF17 and Maelstrom are strongly stained in the SB [19,21]. DDX4 [11] and DDX25 [22] are also stained in this structure but very weakly. The present study showed that the SB was weakly labeled for BRUNOL2. The functions of BRUNOL2 in the cytoplasm are suggested to be the regulation of translation and mRNA silencing [3]. The present study suggests that nuage structures cited above might be the sites for these functions and BRUNOL2 belongs to one of nuage proteins.

The present study demonstrated that BRUNOL2 was localized in several subcellular structures such as ERattached small granules, neck material, reticulated body, MAG, granulated body, and manchette. The most of these structures have shown to be positively stained for DDX25 [22]. The ER-attached small granules seem to be identical to "puffs" described in the cytoplasm of steps 8 -9 spermatids, which are suggested to be assembled to granulated body [23]. The granulated body is composed of polypeptides destined for the formation of the outer 
dense fiber of flagellum [24]. The reticulated body consists of anastomosed cords and is observed in the cytoplasm of steps 14-16 spermatids. The nature and function of are unclear. The MAG appears in the cytoplasm of step 17 spermatids and disappears in step 18 spermatids [23]. The function of this structure is unknown. The ribosome aggregate is composed of numerous free ribosomes and observed in the cytoplasmic lobe of step 19 spermatids and in residual body. The strongest signal for BRUNOL2 is detected in this structure. It seems that some population of BRUNOL2 might be tightly bound to individual free ribosome during spermatogenesis, which is eventually assembled closely into large aggregates, so that the labeling intensity is enhanced as observed. The association of BRUNOL2 with free and membrane-bound ribosomes is observed in somatic cells such as fibroblasts and smooth muscle cells (unpublished data). Manchette is a cytoskeletal complex composed of a sleeve of microtubules and may function in part in alteration of nuclear shape and transport of materials to spermatid head, centrosome and tail [25]. Several proteins interacting with manchette microtubules, including Sak57/K5 [26], Polaris [27] TBP-1 [28], and motor proteins [29,30] have been found in spermatids. The present study suggests that BRUNOL2 may interact directly or indirectly with microtubules.

The present study showed that the major distribution sites of BRUNOL2 were the cytoplasm of early to late spermatocytes and round spermatids and the nuclei of spermatogonia to round spermatids. Such localization seems to match to the functions reported, which are the regulation of transcription [3]. In the nucleus, BRUNOL2 was shown to be closely associated with heterochromatin but not with euchromatin. Transcription is carried out on perichromatin fibrils which extend from heterochromatin $[31,32]$. BRUNOL2 might be concerned with the activation of silenced genes at heterochromatin. In addition, the existence of BRUNOL2 in the XY body and the adjacent nucleolus suggests some function of BRUNOL2 concerned with the XY body.

\section{Acknowledgements}

The work was supported by the University research fund, in part by a grant-in-aid (17570158) from the Ministry of Education, Science, Culture and Sport, and by the Science Research Promotion Fund from the Promotion and Mutual Aid Corporation for Private Schools of Japan.

\section{REFERENCES}

[1] A. N. Ladd, N. Charlet-B. and T. A. Cooper, "The CELF Family of RNA Binding Proteins Is Implicated in Cell-Specific and Developmentally Regulated Alternative Splicing," Molecular and Cellular Biology, Vol. 21, No.
4, 2001, pp. 1285-1296. doi:10.1128/MCB.21.4.1285-1296.2001

[2] H. Suzuki, Y. Jin, H. Otani, K. Yasuda and K. Inoue, "Regulation of Alternative Splicing of $\alpha$-Actinin Transcript by Bruno-Like Proteins," Genes to Cells, Vol. 7, No. 2, 2002, pp. 133-141.

doi:10.1046/j.1356-9597.2001.00506.x

[3] C. Barreau, L. Paillard, A. Méreau and H. B. Osborne, "Mammalian CELF/Bruno-Like RNA-Binding Proteins: Molecular Characteristics and Biological Functions," Biochimie, Vol. 88, No. 5, 2006, pp. 515-525.

[4] L. T. Timchenko, J. W. Miller, N. A. Timchenko, D. R. DeVore, K. V. Datar, L. Lin, R. Roberts, C. T. Caskey and M. S. Swanson, "Identification of a $(\mathrm{CUG})_{n}$ Triplet Repeat RNA-Binding Protein and Its Expression in Myotonic Dystrophy," Nucleic Acids Research, Vol. 24, No. 22, 1996, pp. 4407-4414. doi:10.1093/nar/24.22.4407

[5] X. H. Lu, N. A. Timchenko and L. T. Timchenko, "Cardiac Elav-Type RNA-Binding Protein (ETR-3) Binds to RNA CUG Repeats Expanded in Myotonic Dystrophy," Human Molecular Genetics, Vol. 8, No. 1, 1999, pp. 53-60. doi: $10.1093 / \mathrm{hmg} / 8.1 .53$

[6] R. Roberts, N. A. Timchenko, J. W. Miller, S. Reddy, C. T. Caskey, M. S. Swanson and L. T. Timchenko, "Altered Phosphorylation and Intracellular Distribution of a $(\mathrm{CUG})_{n}$ Triplet Repeat RNA-Binding Protein in Patients with Myotonic Dystrophy and in Myotonin Kinase Knockout Mice," Proceedings of the National Academy of Sciences of the United States of America, Vol. 94, No. 24, 1997, pp. 13221-13226. doi:10.1073/pnas.94.24.13221

[7] A. N. Ladd and T. A. Cooper, "Multiple Domains Control the Subcellular Localization and Activity of ETR-3, a Regulator of Nuclear and Cytoplasmic RNA Processing Events," Journal of Cell Science, Vol. 117, No. 16, 2004, pp. 3519-3529. doi:10.1242/jcs.01194

[8] J. Wu, C. Li, S. Zhao and B. Mao, "Differential Expression of the Brunol/CELF Family Genes during Xenopus Laevis Early Development," The International Journal of Experimental Biology, Vol. 54, 2010, pp. 209-214. doi:10.1387/ijdb.082685jw

[9] L. T. Timchenko, E. Sailsbury, G.-L. Wang, H. Nguyen, J. H. Albrecht, J. W. B. Hershey and N. A. Timchencko, "Age-Specific CUGBP -eIF $_{2}$ Complex Increases Translation of CCAAT/Enhancer-Binding Protein $\beta$ in Old Liver," The Journal of Biological Chemistry, Vol. 281, No. 43, 2006, pp. 32806-32819. doi:10.1074/jbc.M605701200

[10] C. Kress, C. Gautier-Courteille, H. B. Osborne, C. Babinet and L. Pillard, "Inactivation of CUG-BP1/CELF1 Causes Growth, Viability, and Spermatogenesis Defects in Mice," Molecular and Cellular Biology, Vol. 27, No. 3, 2007, pp. 1146-1157. doi:10.1128/MCB.01009-06

[11] Y. Onohara, T. Fujiwara, T. Yasukochi, M. Himeno and S. Yokota, "Localization of Mouse Vasa Homolog Protein in Chromatoid Body and Related Nuage Structures of Mammalian Spermatogenic Cells during Spermatogenesis," Histochemistry and Cell Biology, Vol. 133, No. 6, 2010, pp. 627-639. doi:10.1007/s00418-010-0699-5 
[12] C. de Roe, P. J. Courtoy and P. Baudhuin, "A Model of Protein Colloidal Gold Interactions," Journal of Histochemistry \& Cytochemistry, Vol. 35, No. 11, 1987, pp. 1191-1198. doi:10.1177/35.11.3655323

[13] L. D. Russell, R. A. Ettlin, A. S. P. Hikim and E. D. Clegg, "Histological and Histopathological Evaluation of Testis," Cache River Press, Clearwater, 1990.

[14] Y. Toyooka, N. Tsunekawa, Y. Matsui, M. Satoh and T. Noce, "Expression and Intracellular Localization of Mouse Vasa-Homologue during Germ Cell Development," Mechanisms of Development, Vol. 93, No. 1-2, 2000, pp. 139-149. doi:10.1016/S0925-4773(00)00283-5

[15] T. Noce, S. Okamoto-Ito and N. Tsunekawa, "Vasa Homolog Genes in Mammalian Germ Cell Development," Cell Structure and Function, Vol. 26, No. 3, 2001, pp. 131-136. doi:10.1247/csf.26.131

[16] C.-H. Tsai-Morris, Y. Sheng, E. Lee, K.-J. Lei and M. L. Dufau, "Gonadotropin-Regulated Testicular RNA Helicase (GRTH/Ddx25) is Essential for Spermatid Development and Completion of Spermatogenesis," Proceedings of the National Academy of Sciences of the United States of America, Vol. 101, No. 17, 2004, pp. 6373-6378. doi:10.1073/pnas.0401855101

[17] S. Chuma, M. Hiyoshi, A. Yamamoto, M. Hosokawa, K. Takamune and N. Nakatsuji, "Mouse Tudor Repeat1(MTR-1) is a Novel Component of Chromatoid Bodies/Nuages in Male Germ Cells and Forms a Complex with snRNPs," Mechanisms of Development, Vol. 120, No. 9, 2003, pp. 970-990.

[18] M. Hosokawa, M. Shoji, K. Kitamura, T. Tanaka, T. Noce, S. Chuma and N. Nakatsuji, "Tudor-Related Protein TDRD 1/MTR-1, TDRD6 and TDR7/TRAP: Domain Composition, Intracellular Localization, and Function in Male Germ Cells in Mice," Developmental Biology, Vol. 301 , No. 1,2007 , pp. 38-52. doi:10.1016/j.ydbio.2006.10.046

[19] J. Pan, M. Goodheart, S. Chuma, N. Nakatsuji, D. C. Page and P. J. Wang, "RNF17, a Component of the Mammalian Germ Cell Nuage, is Essential for Spermiogenesis," Development, Vol. 132, 2005, pp. 4029-4039. doi: $10.1242 /$ dev.02003

[20] N. Kotaja, H. Lin, M. Parvienen and P. Sassone-Corsi, "Interplay of PIWI/Argonaute Protein MIWI and Kinesin KIF17b in Chromatoid Bodies of Male Germ Cells," Journal of Cell Science, Vol. 119, No. 119, 2006, pp. 2819-2825. doi: $10.1242 /$ jcs. 03022

[21] S. F. C. Soper, G. W. van der Heijden, T. C. Hardiman, M. Goodheart, S. L. Marten, P. de Boer and A. Bortvin, "Mouse Maelstrom, a Component of Nuage, is Essential for Spermatogenesis and Transposon Repression in Meiosis," Developmental Cell, Vol. 15, No. 2, 2008, pp. 285-297. doi:10.1016/j.devcel.2008.05.015

[22] Y. Onohara and S. Yokota, "Expression of DDX25 in Nuage Components of Mammalian Spermatogenic Cells: Immunofluorescence and Immunoelectron Microscopic
Study," Histochemistry and Cell Biology, Vol. 137, No. 1, 2011, pp. 37-51.

[23] Y. Clermont, R. Oko and L. Hermo, "Cell Biology of Mammalian Spermatogenesis," In: C. Desjardins and L. L. Ewing, Eds., Cell and Molecular Biology of the Testis, Oxford University Press, New York, 1993, pp. 332-376.

[24] Y. Clermont, R. Oko and L. Hermo, "Immunocytoche mical Localization of Proteins Utilized in the Formation of Outer Dense Fibers and Fibrous Sheath in Rat Spermatids: An Electron Microscopic Study," The Anatomical Record, Vol. 227, No. 4, 1990, pp. 447-457. doi:10.1002/ar.1092270408

[25] A. L. Kierszenbaum, "Intramanchette Transport (IMT): Managing the Making of the Spermatid Head, Centrosome, and Tail," Molecular Reproduction and Development, Vol. 63, No. 1, 2002, pp. 1-4. doi: $10.1002 / \mathrm{mrd} .10179$

[26] L. L. Tres and A. L. Kierszenbaum, "Sak57, an Acidic Keratin Initially Present in the Spermatid Manchette before Becoming a Compound of Paraaxonemal Structures of the Developing Tail," Molecular Reproduction and Development, Vol. 44, No. 3, 1996, pp. 395-407. doi:10.1002/(SICI)1098-2795(199607)44:3<395::AID-M RD13>3.0.CO;2-\#

[27] P. D. Taulman, C. J. Hycraft, D. F. Balkovetz and B. K. Yoder; "Polaris, a Protein Involved in Left-Right Axis Patterning, Localizes to Basal Bodies and Cilia," Molecular Biology of the Cell, Vol. 12, No. 3, 2001, pp. 589-599.

[28] E. Rivkin, E. B. Cullinan, L. L. Tres and A. L. Kierszenbaum, "A Protein Associated with the Manchette during Rat Spermiogenesis is Encoded by a Gene of the TBP-1-Like Subfamily with Highly Conserved ATPase and Protease Domain," Molecular Reproduction and Development, Vol. 48, No. 1, 1997, pp. 77-89. doi:10.1002/(SICI)1098-2795(199709)48:1<77::AID-MR D10>3.0.CO;2-T

[29] A. Junco, B. Bhullar, H. A. Tarnasky and F. A. van der Hoorn, "Kinesin Light Chain KLC3 Expression in Testis is Restricted to Spermatids," Biology of Reproduction, Vol. 64, No. 5, 2001, pp. 1320-1330. doi:10.1095/biolreprod64.5.1320

[30] M. G. Miller, D. J. Mulholand and W. A. Vogt, "Rat Testis Motor Proteins Associated with Spermatid Translocation (Dynein) and Spermatid Flagella (Kinesin-II)," Biology of Reproduction, Vol. 60, No. 4, 1999, pp. 1047-1056. doi:10.1095/biolreprod60.4.1047

[31] D. L. Spector, "Nuclear Organization and Gene Expression," Experimental Cell Research, Vol. 229, No. 2, 1996, pp. 189-197. doi:10.1006/excr.1996.0358

[32] M. Labrador and V. G. Corces, "Setting the Boundaries of Chromatin Domains and Nuclear Organization," Cell, Vol. 111, No. 2, 2002, pp. 151-154. doi:10.1016/S0092-8674(02)01004-8 